\title{
Management Strategies for COVID-19 in the General Ward of Cardiovascular Surgery: Experience From a Single Tertiary Hospital in China
}

\author{
Siyi He, ${ }^{1}$ Xiaoyan Liu, ${ }^{1}$ Xia Li, ${ }^{1}$ Xiumei Gao, ${ }^{1}$ Yushan Liu, ${ }^{1}$ Liang Hu,,${ }^{1}$ Lu Chen, ${ }^{1}$ Hui Jiang, ${ }^{1}$ \\ Jinbao Zhang, ${ }^{1}$ Jingwen $\mathrm{Li}^{2}$ \\ ${ }^{1}$ Department of Cardiovascular Surgery, General Hospital of Western Theater Command, Chengdu, China; \\ ${ }^{2}$ Department of Hospital-Acquired Infection Management, West China Hospital, Sichuan University, Chengdu, China
}

\section{ABSTRACT}

Coronavirus disease 2019 (COVID-19) is a highly contagious respiratory disease that threatens global health. During the pandemic period of COVID-19, the task for prevention in the general ward of cardiovascular surgery is fairly arduous. The present study intends to summarize our experience with infection control, including ward setting, admission procedures, personnel management, health education, and so on, to provide reference for clinical management.

\section{INTRODUCTION}

Since December 2019, cases of viral pneumonia or lung infection have been detected around the world. This virus was named "Coronavirus Disease 2019 (COVID-19)" by the World Health Organization on 11 February 2020 [Hu 2021; Dhama 2020]. With the spread of the epidemic, the task for prevention and control is arduous. All regions have introduced local prevention and control policies, in accordance with national laws and regulations to prevent the spread of the virus. Although a variety of COVID-19 vaccines have been available and widely inoculated in the population [Creech 2021; Su 2021], outbreaks still are occurring in many places. At the same time, multiple variant strains have been found in the United Kingdom, India, and Guangzhou, China, thereby the possibility of a new round of global pandemic cannot even be ruled out [Kirby 2021; Callaway 2021]. As the forefront of epidemic prevention and control, hospitals have always been the most critical place for infection management.

Received Fuly 20, 2021; received in revised form August 2, 2021; accepted August 3, 2021.

Correspondence: Finbao Zhang, Department of Cardiovascular Surgery, General Hospital of Western Theater Command, Rongdu Avenue No. 270, Finniu District, Chengdu City, Sichuan Province, China, 610083, Telephone +86-028-86570359 (e-mail: jinbaozhang001@163.com) or Fingwen Li, Department of HospitalAcquired Infection Management, West China Hospital, Sichuan University, Guoxue Lane No. 37, Wubou District, Chengdu City, Sichuan Province, China, 610083, Telephone+86-028-85421969 (e-mail: lijw689@126.com).
This study intends to summarize the strategies for epidemic prevention and control in the general ward of cardiovascular surgery in our hospital, so as to provide reference for clinical management.

\section{PATIENTS AND METHODS}

Characteristics of patients in the department of cardiovascular surgery: The unique clinical characteristics of patients in the department of cardiovascular surgery bring new challenges for the prevention and control of this epidemic. First, the immunity in patients undergoing cardiac surgery, especially cardiopulmonary bypass, will decline to various degrees, so they are more susceptible to pneumonia [Deng 2020]. Second, patients are unable to take care of themselves in the early postoperative period, so they need assistance to help them buy meals, daily goods, etc. Therefore, stricter requirements on personnel management are needed. Third, patients in the early postoperative stage may have fever, dyspnea, hypoxemia and other symptoms, accompanied by pulmonary invasive imaging findings, which are consistent with the initial clinical manifestations of COVID19. Hence, it is difficult for the early diagnosis and differential diagnosis of COVID-19.

Ward settings and disinfection: Special screening posts should be set up at the entrance of general wards. All persons entering the ward should wear surgical masks and disinfect their hands. At the same time, temperature monitoring is conducted and epidemiological history is asked. Patients with normal temperatures and no epidemiological history may enter the ward. Access control of the ward should be strengthened. Irrelevant personnel strictly are prohibited. A COVID-19 isolation ward and special transit channel for suspected patients should be established. The central air conditioning in the isolation ward is turned off. Medical staff should wear protective clothing and goggles when entering the isolation ward.

Windows in the ward are open for ventilation twice a day with duration of at least 30 minutes each time. Treatment rooms or other empty rooms are disinfected with ultraviolet light for 30 minutes daily. Surfaces and floors are disinfected twice daily with a chlorine-containing disinfectant at a concentration of $1000 \mathrm{mg} / \mathrm{L}$. Each bed is equipped with a fixed stethoscope and other medical instruments, which will 
be disinfected after use. A bottle of disinfectant is placed at the end of each bed, and hands will be disinfected immediately after contact with the patient. All waste in the isolation ward is treated as medical waste and placed in double yellow garbage bags, which will be sprayed with chlorine-containing disinfectant. The disinfection registration system strictly is implemented. The head nurse supervises and checks the disinfection work every day.

Admission management: All patients and their escorts must have nucleic acid test results within three days prior to admission and have no contact history with the epidemic area or patients with COVID-19. Suspected patients or patients with no nucleic acid test results should be admitted to the isolation ward through special channels. When the nucleic acid test results are normal, the patient will be transferred to the general ward. Chest CT examination routinely is performed in all patients after admission. When the patients and their escorts are admitted to our department, the nurse in charge of the ward will carry out the education and inform the prevention and control regulations of COVID-19 in detail.

Personnel management - management of patients: Indications for hospitalization of patients should be strictly approved. In principle, patients are not allowed to leave the ward. If they really need to go out, wristbands are necessary. Visits between rooms are forbidden. Body temperature of all personnel in the general ward should be monitored and recorded three times a day. In case of fever, we should evaluate the possibility of COVID-19 based on the results of other ancillary examinations. Suspected patients should be accompanied to the fever clinic in time. Meals of patients are sent to the ward uniformly from the hospital canteen. Unnecessary examinations are eliminated to limit outings. Before surgery, the patient's condition is evaluated by the physician in charge and the anesthesiologist together. Total length of stay should be minimized to the greatest extent. The time of revisit and follow up can appropriately be lengthened. The Internet hospital and other ways can be used for remote diagnosis and treatment to reduce the aggregation of patients.

Management of escorts: In principle, only one caregiver can be assigned to each patient. If the infant patient really needs two caregivers, it shall be agreed by the doctor in charge. Escort cards can be applied for only with nucleic acid test results within three days and with approvement of the head nurse. The primary nurse registers the personal information of the escort in detail, including ID card, telephone number, address, epidemiological history, etc. Escorts can enter or leave the ward only with their escort cards and ID cards. The escorts are relatively fixed and cannot be replaced at will. Escort cards are valid for 14 days and need to be applied for again after the expiry date. Escort cards shall be recovered and destroyed upon discharge from the hospital. The body temperature of the escorts is measured and recorded three times a day. Visiting patients is strictly prohibited in the general ward. Medical staff should guide and encourage family members to visit and greet patients online through video, telephone, and other means. The explanation for illness and preoperative signature with other family members are arranged in the reception room outside the ward.

Management of medical staff: All medical staff shall monitor their own temperature daily and report the results to the head nurse. Once fever or respiratory symptom occurs, it should be reported to the department of hospital infection control. Staff can enter or leave the ward only with their employee's cards. Doctors and nurses carry out nucleic acid tests for COVID-19 every two weeks. When performing routine medical procedures on patients, gloves and disposable surgical gowns should be worn. Working clothes should be kept in designated areas and cleaned weekly. Training and examinations are conducted every month, including policy requirements, diagnosis and treatment of COVID-19, prevention and control of nosocomial infection, etc. Working, living, and dining places for medical staff should be completely separate from patients.

Health education: Management of health publicity and education for patients in the general ward shall be strengthened. The nurse in charge of the bed instructs the patients and their escorts to strengthen self-observation. In case of cough, fever, and other symptoms, they should inform the medical staff on their own initiative and immediately be transferred to the fever clinic. Education brochures are distributed to patients and escorts to give a comprehensive understanding of COVID-19. All personnel should fully know the protection methods for COVID-19, as well as laws and regulations. Fears of every patient should be eliminated as much as possible so they face surgery in a relaxed state of mind. Medical workers are on the frontline of epidemic prevention and control, so they are under great psychological pressure. Attention should be focused on the mental health status of doctors and nurses.

\section{RESULTS}

This standardized protocol was conducted since April 2020 and constantly has been modified and improved, according to the development of the epidemic. In our department, there are 50 beds in general wards. Until July 2021, a total of 801 inpatients were admitted, and 712 surgeries were performed. Through proper management and control, no patients, escorts or medical staff have been infected with COVID-19.

\section{CONCLUSION}

Although the COVID-19 epidemic has gradually entered a stable stage, the prevention and control of infection cannot be slackened. Large mobility of hospital people, strong transmission of virus, and low immunity of patients after cardiac surgery all present severe challenges to the management of the general ward. Through the optimization of ward setting, personnel management, health education and admission procedures, the spread of COVID-19 can effectively be prevented. The above measures deserve widespread promotion during the COVID-19 pandemic. 


\section{REFERENCES}

Callaway E. 2021. Delta coronavirus variant: scientists brace for impact. Nature.

Creech CB, Walker SC, Samuels RJ. 2021. SARS-CoV-2 Vaccines. Jama 325(13):1318-20.

Deng Y, Hou L, Xu Q, et al. 2020. Cardiopulmonary Bypass Induces Acute Lung Injury via the High-Mobility Group Box 1/Toll-Like Receptor 4 Pathway. Disease markers 2020:8854700.
Dhama K, Khan S, Tiwari R, et al. 2020. Coronavirus Disease 2019COVID-19. Clinical microbiology reviews 33(4).

Hu B, Guo H, Zhou P, Shi ZL. 2021. Characteristics of SARS-CoV-2 and COVID-19. Nature reviews Microbiology 19(3):141-54.

Kirby T. 2021. New variant of SARS-CoV-2 in UK causes surge of COVID-19. The Lancet Respiratory medicine 9(2):e20-e1.

Su S, Du L, Jiang S. 2021. Learning from the past: development of safe and effective COVID-19 vaccines. Nature reviews Microbiology 19(3):211-9. 